Original Research Paper

\title{
An Examination of Factor Structure of the Hamilton Anxiety Rating Scale in a Non-Clinical Persian Sample
}

\author{
${ }^{1}$ Paul Slater, ${ }^{2}$ Brendan Bunting, ${ }^{1}$ Felicity Hasson, ${ }^{3}$ Ahmed Mohammad Al-Smadi, \\ ${ }^{3}$ Omar Salem Gammouh, ${ }^{4}$ Ala Ashour and ${ }^{5}$ Damian Jordan \\ ${ }^{1}$ Institute of Nursing and Health Research, Ulster University, Coleraine, Northern Ireland \\ ${ }^{2}$ Psychology Research Institute, Ulster University, Coleraine, Northern Ireland \\ ${ }^{3}$ Faculty of Health Science, American University of Madaba, Jordan \\ ${ }^{4}$ Faculty of Nursing, Hashemite University, Zarqa Jordan \\ ${ }^{5}$ Belfast Health and Social Care Trust, Northern Ireland
}

Article history

Received: 22-02-2019

Revised: 28-03-2019

Accepted: 20-05-2019

Corresponding Author:

Paul Slater

Institute of Nursing and

Health Research, Ulster

University, Coleraine,

Northern Ireland

Email: pf.slater@ulster.ac.uk

\begin{abstract}
The Hamilton Anxiety Rating Scale is an internationally used assessment tool for anxiety level however its acceptable factor structure remains fluid. The aim of this paper is to examine the psychometric properties and establish normative anxiety prevalence rates with a Persian randomized sample. A cross sectional survey was distributed to a randomised sample of adults $(n=1260)$ in the Middle East. A two-factor model was deemed the most statistically and theoretically appropriate measurement model. This represented a split between cognitive and physiological symptoms. Overall, $15.2 \%$ of respondents reported at least mild anxiety, with $2.3 \%$ experiencing severe anxiety. Lower anxiety scores on the scale were reported among males, the married, younger and higher educated people. The Hamilton Anxiety Scale continues to be a useful tool in the measurement of anxiety. The two factor model separates anxiety into two dimensions, cognitive and physiological aspects and both are highly correlated with each other suggesting a single overarching construct of anxiety. The two-factor model may help provide a better understanding of the effectiveness of different therapeutic techniques. The pooled prevalence of ADs in a non-clinical Persian sample was relatively lower than those of some other countries.
\end{abstract}

Keywords: Anxiety, Hamilton Anxiety Rating Scale, Normative Data, Psychometrics

\section{Introduction}

Anxiety Disorders (ADs) are common psychiatric conditions affecting an estimated $10 \%$ of the global population (Alonso et al., 2018). ADs include panic disorders with or without agoraphobia, selective mutism, specific phobias, generalized anxiety disorder, social anxiety disorder and separation anxiety disorder DSM-V (APA, 2013).

Prevalence rates across the world range from $24.47 \%$ in China (Guo et al., 2016), 18\% in the United States (Kessler and Wang, 2008), 13.6\% in Europe (Alonso and Lepine, 2007) and in $8.1 \%$ in Japan (Ishikawa et al., 2015). Several studies have reported a higher prevalence for females (Remes et al., 2016), lower education levels (Kawakami et al., 2012), younger age groups (Lenze and
Wetherell, 2011; Baxter et al., 2013) and marital status (Kessler et al., 2005; Scott et al., 2010).

ADs have far reaching social, economic and health burden consequences. For example, a global survey associated ADs with risk to education, income and suffering multiple pains Gureje et al., 2008; Levinson et al., 2010), with immense health care costs (Bandelow and Michaelis, 2015). Despite the significant problems caused by anxiety, it is well documented that it is poorly diagnosed and inadequately treated (Bystritsky et al., 2013).

However adequate treatment of anxiety disorders begins with early detection and diagnoses. The accurate screening for anxiety disorders is the starting point for therapeutic intervention and currently there exists a range of tools available. One widely used DSM based anxiety; questionnaire is the Hamilton Anxiety Rating 
Scale (HAM-A sometimes referred to as HADS) (Hamilton, 1959; Thompson, 2015). The 14 items in this measure were originally developed to detect the presence and severity of anxiety and depression in clinical populations. Created prior to modern classification systems such as the DSM-V (APA, 2013) it has formed the benchmark for other devised scales (Beck and Steer, 1991; Beck et al., 1998; Donzuso et al., 2014). Whilst several papers have reported good reliability and validity (Maier et al., 1988; Clark and Donvan, 1994; Mondolo et al., 2006; Kummer et al., 2010; Leentjens et al., 2011) with acceptable Cronbach alpha scores were reported in numerous studies (Bjelland et al., 2002; García-Campayo et al., 2012; Chouhan et al., 2016; Erge et al., 2016). However, both Scott et al. (2017) and Litster et al. (2016) reported little support for the validity and reliability of the HAM-A. The factors structure of the instrument is not standardised, influenced by the medical condition being investigated, ranging from one to a four structure as fitting the data equally well (Bjelland et al., 2002; Leentjens et al., 2011; Cosco et al., 2012; Hung et al., 2015; Scott et al., 2017).

Nevertheless, since its development HAM-A has been translated into several languages (Fateem, 1998; Kummer et al., 2010; García-Campayo et al., 2012; Erge et al., 2016; Chouhan et al., 2016) and utilised in a range of clinical conditions (Capuron et al., 2002; Kersting et al., 2004; Gençöz et al., 2007; Garalejić et al 2010; Kummer et al., 2010; Chouhan et al., 2016; Erge et al., 2016; Yang et al., 2017).

Whilst ADs are some of the most common psychiatric conditions in the Western world (Simpson et al., 2010) few studies have reported the prevalence of anxiety in Arab countries in non-clinical adult populations (Karam et al., 2008; Bahammam, 2016) despite exposure to war and conflict existing in the region (Tanios et al., 2009) which Baxter et al. (2013) reports has a substantial impact of the prevalence of anxiety disorders. In a review paper of anxiety among adults in Saudi Arabia reported prevalence rates ranging from $7-33 \%$, with gender differences detected (Tanios et al., 2009). However, the psychometric information regarding the Arabic version of the HAM-A is limited to studies of specific clinical populations (El-Rufaie and Absood 1995; Hassan et al., 2015; Terkawi et al., 2017). Given the HAM-A continued use in clinical practice and in research, there is a need to identify and, furthermore, test the factor structure of the HAM-A with a normative population to establish what exactly it is measuring. Moreover, as definitions and classifications of anxiety change, new research emerges and new technologies advance the knowledge base of symptomology relating to anxiety, there is a need to ensure that measurement tools such as the scale stay relevant as effective measures in todays' society (Leichsenring, 2006; Leentjens et al., 2008). The aim of the current study was to extend this research to explore the psychometric properties of the HAM-A in a non-clinical population in Saudi Arabia.

\section{Methods}

\section{Participants}

A large scale quantitative, cross sectional survey was conducted with a randomly selected sample of 1,500 individuals living in Amman; the capital city of Jordan as part of a study to look at COPD and psychological comorbidities (Al-Smadi et al., 2017) and focused on people over 40 years of age. Participants were recruited and assessed using HAM-A Scale and key demographic details also recorded.

\section{Procedure}

The sampling frame consisted of a list of registered residents living in Amman, obtained from Amman Municipality authorities. A sampling frame of 200,000 houses in total. A random sample of 1500 houses that selected using a random number generator and were identified for inclusion on the study. Researcher assistants trained in the collection of psychological data conducted all interviews face-to face. Of the 1500 houses approached, 90 houses approached included individuals who were less than 40 years old and therefore excluded. In total, 150 individuals refused to participate in the study without explanation. A total sample comprised of 1260 participants drawn from Jordan and Iraq representing a response rate of $84 \%$. Based on a potential sampling frame of 20,000 houses and 2 adults per house and with $95 \%$ confidence level and 50\% anticipated response accuracy, the sample size represents confidence interval of 2.72 (www.surveysystem.com/sscalc.htm).

\section{Measure}

The Hamilton Anxiety Rating Scale (HAM-A, Hamilton (1959) is a 14 items scale designed to assess the individuals level of both psychic and somatic anxiety, measured on a 5-point Likert scale ranging from 0 (not present) and 4 (Severe). Total scores range from 0-56. The tool was translated into Arabic by Fateem (1998) and its psychometric properties of the tool have been demonstrated with an Arabicspeaking sample (Gammoh et al., 2016) and wider afield (Maier et al., 1988; Kummer et al., 2010; Leentjens et al., 2011). Demographic details collected were also presented (Table 1).

\section{Analytic Plan}

Descriptive and measures of dispersion statistics were generated for all items to help inform subsequent analysis. Measures of appropriateness to conduct factor analysis was conducted using The Kaiser-Meyer-Olkin 
Measures of Sampling Adequacy and Bartletts test of sphericity. Inter-item correlations were generated to examine for collinearity prior to full analysis. Exploratory factor analysis was conducted using maximum likelihood extraction comparing a 1-4 factor model to identify the most statistically appropriate model. A theoretically derived model based on the results from the exploratory factor analysis was then analysed within a confirmatory framework, using the same data. Once a model had been identified, confirmatory factor analysis was conducted and the model fitted.

The model was re-specified using the modification indices provided in the statistical output until acceptable and a statistically significant relationship identified. All re-specifications of the model were guided by principles of meaningfulness (a clear theoretical rationale); Transitivity (if A is correlated to B and B correlated to $\mathrm{C}$, then A should correlate with $\mathrm{C}$ ); Generality rule if there is a reason for correlating the errors between one pair of errors, then all pairs for which that reason applies should also be correlated (Kenny, 2011).

Acceptance modification criteria of:

1. The items to first order factors were fitted initially

2. Correlated error variance permitted as all items were measuring the same unidimensional construct

3. Only statistically significant relationship retained to help produce as parsimonious a model as possible

4. Factor loadings above 0.45 to provide a strong emergent factor structure

Acceptable fit statistics were set at Root Mean Square Estimations of Approximation (RMSEA) of 0.05 or below; 90\% RMSEA higher bracket below 0.08; and Confirmation Fit Indices (CFI) of 0.95 or higher and SRMR below 0.05 (Byrne, 2011; Wang and Wang, 2012). Cronbach alpha scores were generated for factors in the accepted factor model. The impact of demographic details on the factor model was examined using regression.

\section{Results}

There was an even distribution of male and female respondents in the sample. The majority $(92.9 \%)$ of participants were Jordanians and married (78.0\%; Table 1 ). Most of the participants were aged 40-49 years of age and had a degree.

\section{Measures of Distribution}

Mean scores ranged from 0.322 (behaviour at interview) indicating 'not present' to 1.079 'mild' (Tension). Two items (Cardiovascular symptoms and Behaviour on interview) were scored low, with a floor effect and positively skewed accordingly. Seven items had scores that exceeded acceptable boundaries of kurtosis (Fears, Cardiovascular Symptoms, Respiratory Symptoms, Gastrointestinal Symptoms, Genitourinary Symptoms, Autonomic Symptoms, Behaviour at interview). Items with high kurtosis scores were the items that scored low in anxiety levels (Table 2). Correlation matrix of items shows scores ranged from 0.246 and 0.691 and collinearity was not an issue.

\section{Exploratory Factor Analysis}

The factor model was tested using exploratory factor analysis and a 1-3 factor model examined. The items were treated as categorical and Maximum Likelihood Robust (MLR) estimator used due to the issue of skewness and kurtosis of the items. The Akaike Information Criterion (AIC) and the Bayesian Information Criterion (BIC, called the Schwarz criterion), can also be used to compare models, including non-nested models. All three fit statistics provide information on comparative models and lower values indicate a better fit so the model with the lowest AIC, BIC or adjusted BIC is the best fitting model (Kenny, 2015).

Examination of the factor statistics show significant improvements between a 1-factor and 2-factor model and minimal improvement between a 2-factor and 3-factor model (Table 3). Examination of the emergent factor structure of the three models show the 2-factor model to provide the best fit factor model and simplest theoretical model (Table 4).

Table 1: Demographic details of participants

\begin{tabular}{llll}
\hline Demographic & Percentage & Demographic & Percentage \\
\hline Male & $49.8 \%(\mathrm{n}=627)$ & Less than secondary & $8.3 \%(\mathrm{n}=105)$ \\
Female & $50.2 \%(\mathrm{n}=633)$ & Secondary School & $24.6 \%(\mathrm{n}=310)$ \\
Jordanian & $92.9 \%(\mathrm{n}=1171)$ & Degree & $54.9 \%(\mathrm{n}=692)$ \\
Other & $7.1 \%(\mathrm{n}=89)$ & Post Graduate & $12.1 \%(\mathrm{n}=153)$ \\
Married & $78.0 \%(\mathrm{n}=983)$ & $40-49$ years & $45.1 \%(568)$ \\
Divorced & $6.0 \%(\mathrm{n}=76)$ & $50-59$ years & $30.7 \%(\mathrm{n}=387)$ \\
Widowed & $7.7 \%(\mathrm{n}=97)$ & $60-69$ years & $15.2 \%(\mathrm{n}=191)$ \\
Single & $8.3 \%(\mathrm{n}=104)$ & $70+$ years & $9.0 \%(\mathrm{n}=114)$ \\
\hline
\end{tabular}


Paul Slater et al. / International Journal of Research in Nursing 2019, Volume 10: 1.9 DOI: 10.3844 ijrnsp.2019.1.9

Table 2: Mean scores (SD), Skewness, Kurtosis and factor loading of items of Hamilton anxiety rating scale

\begin{tabular}{llll}
\hline & Mean (SD) & Skewness & Kurtosis \\
\hline Anxious Mood & 1.064 & 0.732 & 0.123 \\
Tension & 1.096 & 0.669 & -0.223 \\
Fears & 0.557 & 1.719 & 2.540 \\
Insomnia & 0.969 & 0.840 & 0.023 \\
Intellectual & 0.940 & 0.985 & 0.355 \\
Depressed Mood & 0.796 & 1.068 & 0.605 \\
Somatic (Muscular) & 1.079 & 0.641 & -0.306 \\
Somatic (Sensory) & 0.573 & 1.498 & 1.766 \\
Cardiovascular Symptoms & 0.416 & 2.126 & 4.328 \\
Respiratory Symptoms & 0.581 & 1.580 & 2.164 \\
Gastrointestinal Symptoms & 0.475 & 1.799 & 2.935 \\
Genitourinary Symptoms & 0.433 & 1.929 & 3.441 \\
Autonomic Symptoms & 0.424 & 1.977 & 3.953 \\
Behaviour at interview & 0.322 & 2.328 & 5.797 \\
\hline
\end{tabular}

Table 3: Comparative fit statistics (EFA with categorical data) for 1-3 factor model of HAM-A

\begin{tabular}{llll}
\hline & Akaike (AIC) & Bayesian (BIC) & Sample-Size Adjusted BIC \\
\hline Factor Model & 32803.772 & 33163.437 & 32941.084 \\
Factor Model & 32368.282 & 32794.742 & 32531.095 \\
Factor Model & 32260.436 & 32748.553 & 32446.789 \\
\hline
\end{tabular}

Table 4: Two-factor (Categorical Data) model of HAM-A

\begin{tabular}{llc}
\hline ITEMs & Factor 1 & Factor 2 \\
\hline Anxious Mood & 0.867 & 0.560 \\
Tension & 0.900 & 0.608 \\
Fears & 0.536 & 0.574 \\
Insomnia & 0.634 & 0.658 \\
Intellectual & 0.520 & 0.624 \\
Depressed Mood & 0.639 & 0.708 \\
Somatic (Muscular) & 0.550 & 0.705 \\
Somatic (Sensory) & 0.463 & 0.704 \\
Cardiovascular Symptoms & 0.471 & 0.713 \\
Respiratory Symptoms & 0.472 & 0.723 \\
Gastrointestinal Symptoms & 0.432 & 0.707 \\
Genitourinary Symptoms & 0.439 & 0.744 \\
Autonomic Symptoms & 0.492 & 0.733 \\
Behaviour at interview & 0.423 & 0.661 \\
\hline
\end{tabular}

\section{Confirmatory Factor Analysis}

Fit statistics for the two-factor model show an acceptable model: RMSEA $=0.058,90 \%$ RMSEA $=$ 0.053-0.064, CFI $=0.919$, SRMR $=0.040$. Suggested modifications include item 2 with Item 1 (Tension with Anxious Moods $=179.376$ ) and cross factor loading cognitive on V7 (Somatic muscular $=48.119$ ). Model 2: RMSEA $=0.041,90 \%$ RMSEA $=0.035-0.047$, CFI $=$ $0.960, \mathrm{SRMR}=0.031$. There was a correlation between cognitive and physiological measures $($ Est $=0.800$, S.E. $=0.021$, Est $/$ S.E. $=37.607)$. All relationships were statistically significant including modifications introduced in the first model. The factor loadings for all items were acceptable (Fig. 1). Item 7 (Somatic muscular) was a cross factor loading and allowed to influence both factors, however examination of the factor loading suggest it loads more strongly to cognitive symptoms.

\section{Corrected Item to Total correlation}

Cronbach's alpha for the total items and the 2 factors were acceptable (Cognitive $=0.84,7$ items; Physiological $=0.81,7$ items; Total scale $=0.89,14$ items). Examination of the alpha if item deleted scores indicate that this score remained static regardless of item deleted. Corrected item-to-total correlations indicating good discrimination (Cognitive $=0.808-0.835$; physiological $=0779-0.786$; Total scale $0.876-0.884$ ).

\section{Impact of Demographic Details on HAM-A}

Fit statistics for the second total sample show an acceptable model: $\mathrm{RMSEA}=0.037,90 \%$ RMSEA $=$ $0.033-0.041, \mathrm{CFI}=0.934, \mathrm{SRMR}=0.028$. Education 
levels had an impact on cognitive anxiety levels were higher levels of education were associated with lower anxiety levels (Degree level education Est $=-0.328$, S.E. $=0.138$, Est/S.E. = -2.369; Postgraduate level education Est $=-0.499$, S.E. $=0.153$, Est $/$ S.E. $=-3.265$ ). Being female was associated with higher levels of anxiety Est = -0.224 , S.E. $=0.065$, Est/S.E. $=-3.441$ ).

The impact of demographic details on physiological symptoms on physiological measure (as shown in Fig. 1), a small but significant relationship was noted between marital status and being widowed (Est $=0.358$, S.E, $=$ 0.162 , Est/S.E. $=2.214$ ) where lower levels of anxiety were noted among married participants. Education levels had an impact on cognitive anxiety levels were higher levels of education were associated with lower anxiety levels (Degree level education Est $=-.519$, S.E. $=0.138$, Est/S.E. = -3.766; Postgraduate level education Est = 0.536 , S.E. $=0.145$, Est $/$ S.E. $=-3.692)$. Being over 70 years of age increased likelihood of anxiety (Est $=0.493$, $\mathrm{S} . \mathrm{E},=0.150$, Est $/$ S.E. $=3.282$ ). All relationships were at a statistically significant level.

\section{Clinical Definition}

Using Hamilton's original categorisation of clinical severity at least $84.8 \%$ of the participants did not display symptoms of clinical anxiety; $2.3 \%$ of the participants reported severe anxiety. Establishing overall prevalence scores allows for the comparison of prevalence across key demographic details. There was a clear gender difference were females reported higher levels of anxiety across all 3 clinical classifications Chi-square 14.689, df $=3, p=0.002$. The relatively small subsample sizes across the other demographic details limits accurate comparisons at a statistically significant level. Cross tabulation shows that education levels have a significant impact on prevalence rates. The more educated the participant the less likely the presence of a clinical diagnosis or the severity of the anxiety. Interestingly, high levels of anxiety were noted among the oldest age group $(70$ years + ). Similarly, high levels of anxiety were noted among the widowed (Table 5).

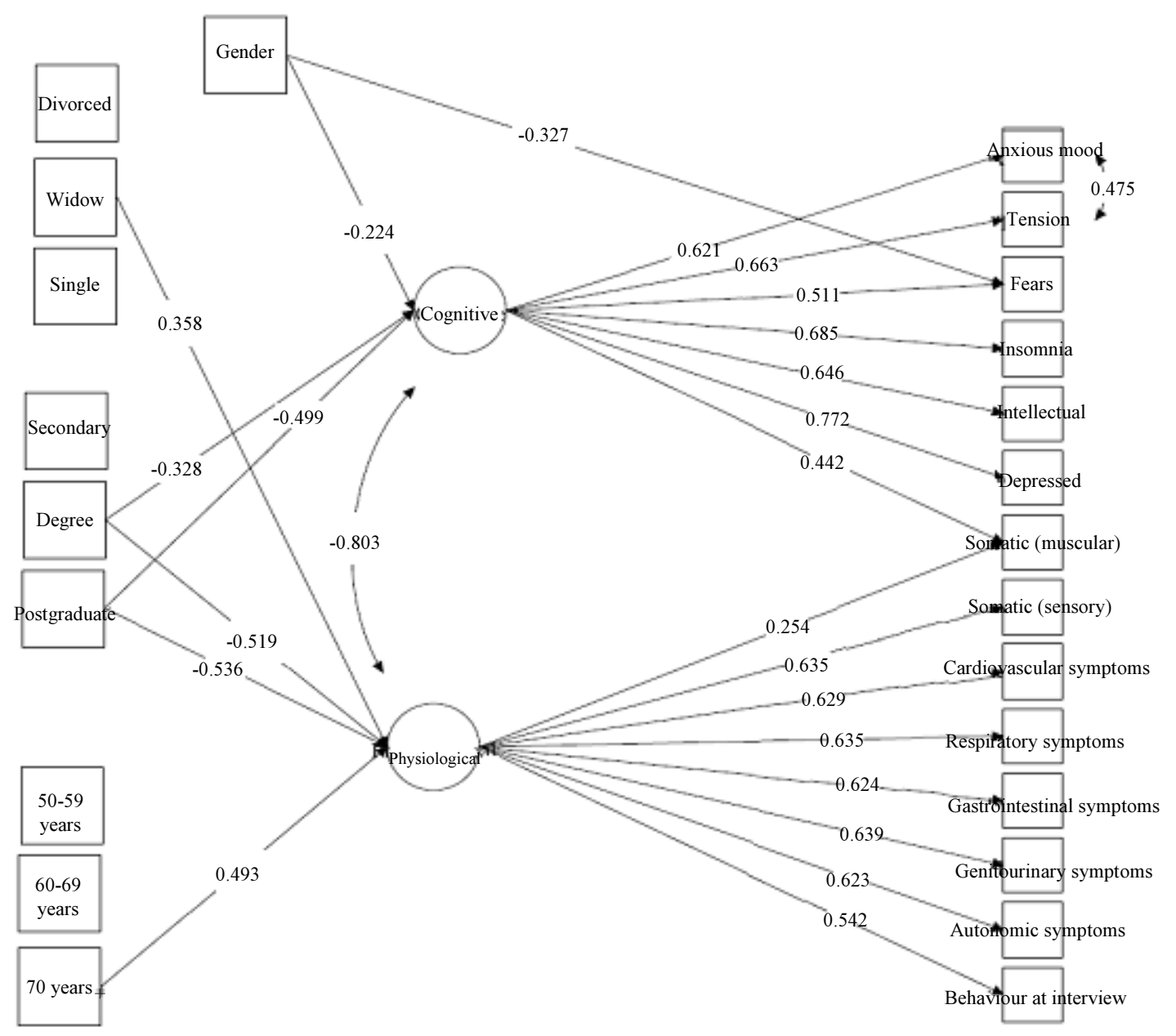

Fig. 1: The measurement model of the HAM-A and statistically significant demographic characteristics 
Table 5: Prevalence of clinical definition of anxiety disorders across definitions

\begin{tabular}{|c|c|c|c|c|}
\hline & None-Mild & Mild-Moderate & Moderate-Severe & Severe \\
\hline Female & $81.8 \%$ & $10.8 \%$ & $3.9 \%$ & $3.5 \%$ \\
\hline Male & $87.8 \%$ & $7.0 \%$ & $4.2 \%$ & $1.0 \%$ \\
\hline Married & $86.7 \%$ & $9.0 \%$ & $3.4 \%$ & $1.4 \%$ \\
\hline Divorced & $89.3 \%$ & $4.0 \%$ & $2.7 \%$ & $4.0 \%$ \\
\hline Widowed & $70.8 \%$ & $12.4 \%$ & $11.2 \%$ & $5.6 \%$ \\
\hline Single & $81.1 \%$ & $8.4 \%$ & $4.2 \%$ & $6.3 \%$ \\
\hline Less than secondary & $66.7 \%$ & $18.3 \%$ & $9.7 \%$ & $5.4 \%$ \\
\hline Secondary School & $79.9 \%$ & $11.2 \%$ & $6.5 \%$ & $2.4 \%$ \\
\hline BS & $88.1 \%$ & $6.7 \%$ & $2.9 \%$ & $2.3 \%$ \\
\hline Post Graduate & $91.4 \%$ & $7.9 \%$ & $0.7 \%$ & $0.0 \%$ \\
\hline 40-49 years & $85.9 \%$ & $9.4 \%$ & $2.6 \%$ & $2.1 \%$ \\
\hline $50-59$ years & $88.6 \%$ & $6.5 \%$ & $3.0 \%$ & $1.9 \%$ \\
\hline $60-69$ years & $82.7 \%$ & $9.5 \%$ & $6.7 \%$ & $1.1 \%$ \\
\hline $70+$ years & $70.0 \%$ & $13.6 \%$ & $10.0 \%$ & $6.4 \%$ \\
\hline Jordanian & $84.5 \%$ & $9.2 \%$ & $4.3 \%$ & $2.1 \%$ \\
\hline Non Jordanian & $89.7 \%$ & $5.1 \%$ & $0.0 \%$ & $5.1 \%$ \\
\hline Overall & $84.8 \%$ & $8.9 \%$ & $4.0 \%$ & $2.3 \%$ \\
\hline
\end{tabular}

\section{Discussion}

The Hamilton Anxiety Rating Scale has been identified as one of the earliest tools to measure the severity of perceived anxiety symptoms (Thompson, 2015) and is one of the most widely used scales in the world (Erge et al., 2016). This study presents acceptable psychometric information relating to an Arabic version of an instrument to measure anxiety. The findings support the use of the HAM-A as an internationally renowned tool for measuring anxiety with wellestablished psychometric properties both here and in previous studies (Beck and Steer, 1991; Mondolo et al., 2006; García-Campayo et al., 2012). However, the findings challenge Leentjens et al. (2011) assertion that a one factor model was a better fit. A two-factor model of anxiety was supported by the study findings and it confirms Hamilton's original 2 factor model of somatic and psychic (Hamilton, 1959). Factor loadings were acceptable and very stringent with such a large sample size and provide surety in the statistical analysis. The Cronbach's alpha scores reported in this study confirm similar scores reported by Leentjens et al. (2011) however with a much larger sample size. The sample sizes in the sample provide an acceptable respondent to item ratio of over 90: 1, well above the 10: 1 as recommended by Hair et al. (2010).

The separation of anxiety into two separate (cognitive and physiological) but correlated constructs may provide a very useful model for the future examination of the impact of interventions for anxiety such as psychopharmacological treatment. The physiological dimension of anxiety would be affected by medication, yet this may not impact on the cognitive dimension of anxiety. It would also provide a better understanding of the impact of psychological 'talking therapies' in isolation or in combination with psychopharmacological interventions. Measuring how different therapeutic techniques impact on different dimensions of anxiety may help provide a better understanding of the effective treatment, identify relapse predictors or help identify when treatments should be discontinued. The high correlation between constructs suggests that all 14 items of the HAM-A are measuring an overarching single item of anxiety. Cronbach alpha scores confirm the summation of items of the HAM-A to produce an overall classification score and clinical banding.

The overall prevalence of anxiety was substantially lower in this study than reported in other western (Alonso and Lepine, 2007; Kessler and Wang, 2008) and Asian (Ishikawa et al., 2015; Guo et al., 2016) countries. The rates in this study do reflect Tanios et al. (2009) 7$33 \%$ range for Persian samples. However, as alluded to by Baxter et al. (2013) the figures reported for this region in the Middle East need to be considered in light of the recently experienced high levels of refugee influx and political unrest, which may account for the 'background anxiety' as exemplified by the level of mild anxiety.

Examination of the results support previous research where higher prevalence rates reported by females (Remes et al., 2016; Baxter et al., 2013), lower education levels (Kawakami et al., 2012) and marital status (Kessler et al., 2005). Interestingly, the findings here reported higher anxiety among participants aged over 70 years old, contrary to previous research (Lenze and Wetherell, 2011; Baxter et al., 2013) which found less anxiety among older people.

\section{Limitations}

Whilst the study's findings are based on a large nonclinical sample size, the methods of sample recruitment and data collection may not ensure sample 
representativeness. Whilst Hamilton's (1959) original theoretical model indicates two distinct categories of psychic and somatic symptoms and this theoretical demarcation is supported in this study, the model requires confirmation across other countries with larger samples as well as more psychometric testing. Moreover the 2-factor model fits the data but with the introduction of correlated errors across items and cross factor loading. This would indicate the presence of additional factors that are not being measured here. Further research is required to identify these factors.

However further examination of the psychometric properties of the HAM-A instrument, the factor structuring of the instrument and the testing of the model in clinical interventions would provide a better understanding of how therapeutic interventions effect different aspects of anxiety. This may prove useful in examining the progression of effective treatment (or not) of anxiety.

\section{Conclusion}

The HAM-A instrument is a valid and reliable instrument for measuring anxiety in an adult population, internationally and across clinical populations. The study also benchmarks the normative levels of anxiety in a Persian non-clinical sample and identifies contributing factors influencing anxiety rates. Interestingly, this study highlights that anxiety levels are higher among older people.

\section{Acknowledgement}

We wish to acknowledge the people of Amman, Jordan for participating in this study.

\section{Funding Information}

This research did not receive any specific grant from funding agencies in the public, commercial, or not-forprofit sectors.

\section{Author's Contributions}

Paul Slater, Felicity Hasson, Brendan Bunting and Damian Jordan: Were responsible for the data analysis, drafting and completion of the manuscript.

Ahmed Mohammad Al-Smadi, Omar Salem Gammouh and Ala Ashour: Were responsible for study design, ethical application and data collection. All authors contributed to the final drafting of the paper.

\section{Ethics}

Full ethical approval was gained prior to commencement of the study and the research was conducted in accordance with the Helsinki Declaration as revised 1989. All principles of good clinical practice in research were adhered to throughout the study. Information sheet was distributed and explained to each potential participant. Consent forms were signed by willing participants. Confidentiality and anonymity of participants' responses were ensured and the data was securely stored in line with current university policy.

\section{References}

Alonso, J. and J.P. Lepine, 2007. Overview of key data from the European Study of the Epidemiology of Mental Disorders (ESEMeD). J. Clin. Psychiatry, 68: 3-90.

Alonso, J., Z. Liu, S. Evans-Lacko, E. Sadikova and G. Thornicroft et al., 2018. Treatment gap for anxiety disorders is global: Results of the world mental health surveys in 21 countries. Depression Anxiety, 35: 195-208. DOI: 10.1002/da.22711

Al-Smadi, A.M., L.I. Tawalbeh, O.S. Gammoh, A. Ashour and F.A. Alzoubi et al., 2017. Predictors of coping strategies employed by Iraqi refugees in Jordan. Clin. Nurs. Res., 26: 592-607.

DOI: $10.1177 / 1054773816664915$

APA, 2013. Diagnostic and Statistical Manual of Mental Disorders DSM-5. 5th Edn., American Psychiatric Pub, ISBN-10: 0890425574, pp: 991.

Bahammam, M.A., 2016. Validity and reliability of an Arabic version of the state-trait anxiety inventory in a Saudi dental setting. Saudi Med. J., 37: 668-674. DOI: $10.15537 / \mathrm{smj} .2016 .6 .13935$

Bandelow, B. and S. Michaelis, 2015. Epidemiology of anxiety disorders in the 21 st century. Dialogues Clin. Neurosco., 17: 327-35.

Baxter, A.J., K.M. Scott, T. Vos and H.A. Whiteford, 2013. Global prevalence of anxiety disorders: A systematic review and meta-regression. Psychol. Med., 43: 897-910. DOI: $10.1017 / \mathrm{S} 003329171200147 \mathrm{X}$

Beck, A.T. and R.A. Steer, 1991. Relationship between the Beck anxiety inventory and the Hamilton anxiety rating scale with anxious outpatients. J. Anxiety Disorders, 5: 213-223. DOI: 10.1016/0887-6185(91)90002-B

Beck, A.T., N. Epstein, G. Brown and R.A. Steer, 1988. An inventory for measuring clinical anxiety: Psychometric properties. J. Consult. Clin. Psychol., 56: 893-897. DOI: 10.1037/0022-006X.56.6.893

Bjelland, I., A.A. Dahl, T.T. Haug and D. Neckelmann, 2002. The validity of the hospital anxiety and depression scale: An updated literature review. J. Psychometric Res., 52: 69-77. DOI: 10.1016/S0022-3999(01)00296-3 
Byrne, B.M., 2011. Structural Equation Modeling with Mplus: Basic Concepts, Applications and Programming. 1st Edn., Routledge Academic, New York.

Bystritsky, A., S. Shalsa, M. Cameron and J. Schiffman, 2013. Current diagnosis and treatment of anxiety disorders. Pharmacy Therapeut., 38: 30-38.

Capuron, L., J.F. Gumnick, D.L. Musselman, D.H. Lawson and A. Reemsnyder et al., 2002. Neurobehavioral effects of interferon- $\alpha$ in cancer patients: phenomenology and paroxetine responsiveness of symptom dimensions. Neuropsychopharmacology, 26: 643.

Chouhan, S.C., P. Singh and S. Kumar, 2016. Assessment of stress and anxiety in parents of children with intellectual disability. Indian J. Health Wellbeing.

Clark, B.D. and J.E. Donovan, 1994. Reliability and validity of the Hamilton anxiety rating scale in an adolescent Sample. J. Am. Acad. Child Adolescent Psychiatry, 33: 354-60. DOI: 10.1097/00004583-199403000-00009

Cosco, T.D., F. Doyle, M. Ward and H. McGee, 2012. Latent structure of the hospital anxiety and depression scale: A 10-year systematic review. J. Psychosom Res., 72: 180-184.

DOI: $10.1016 /$ j.jpsychores.2011.06.008

Donzuso, G., A. Cerasa, M.C. Gioia, M. Caracciolo and A. Quattrone, 2014. The neuroanatomical correlates of anxiety in a healthy population: Differences between the State-Trait anxiety inventory and the hamilton anxiety rating scale. Brain and Behavior., 4: 504-514. DOI: $10.1002 /$ brb3.232

El-Rufaie, O.E. and G.H. Absood, 1995. Retesting the validity of the Arabic version of the Hospital Anxiety and Depression (HAD) scale in primary health care. Soc. Psychiatry Psychiatr. Epidemiol., 30: 26-31. DOI: 10.1007/BF00784431

Erge, D., P. Uysal, H. Aksu, S. Avcil and B.G. Özgür et al., 2016. Psychological status of adolescents with respiratory allergic diseases and their caregivers. Meandros Med. Dental J., 17: 120-128. DOI: $10.4274 /$ meandros. 2630

Fateem, L., 1998. Arabic manual of Hamilton anxiety scale, translated and adapted by lotfy fateem. The Anglo-Egyptian Bookshop. Egypt.

Gammoh, O.S, A.M. Al-Smadi, F.A. Ashour and W. AlAwaida, 2016. Salivary nitric oxide, a biomarker for stress and anxiety? Psychiatry Invest., 13: 311-315. DOI: 10.4306/pi.2016.13.3.311

Garalejić, E., D. Bojović-Jović, A. Damjanović, B. Arsić and I. Pantić et al., 2010. Hamilton Anxiety Scale (HAMA) in infertile women with endometriosis and its correlation with magnesium levels in peritoneal fluid. Psychiatry Danubina, 22: 64-67.
García-Campayo, J., E. Zamorano, M.A. Ruiz, M. PérezPáramo and V. López-Gómez et al., 2012. The assessment of generalized anxiety disorder: psychometric validation of the Spanish version of the self-administered GAD-2 scale in daily medical practice. Health Quality Life Outcomes, 10: 114-114. DOI: $10.1186 / 1477-7525-10-114$

Gençöz, F., T. Gençöz and A. Soykan, 2007. Psychometric properties of the Hamilton depression rating scale and other physician-rated psychiatric scales for the assessment of depression in ESRD patients undergoing hemodialysis in Turkey. Psychiatry Health Med., 12: 450-459. DOI: $10.1080 / 13548500600892054$

Guo, X., Z. Meng, G. Huang, J. Fan and W. Zhou et al., 2016. Meta-analysis of the prevalence of anxiety disorders in mainland China for 2000 to 2015 . Sci. Reports, 6: 28033-28033. DOI: 10.1038/srep28033

Gureje, O., M. Von Korff, L. Kola, K. Demyttenaere and Y. He et al., 2008. The relation between multiple pains and mental disorders: Results from the World Mental Health Surveys. Pain, 135: 82-91.

DOI: 10.1016/j.pain.2007.05.005

Hair, J., B. Black, B. Babin and R. Anderson, 2010. Multivariate Data Analysis. 7th Edn., Pearson Prentice Hall, Upper Saddle River New Jersey.

Hamilton, M., 1959. The assessment of anxiety states by rating. Br. J. Med. Psychol., 32: 50-5. DOI: $10.1111 /$ j.2044-8341.1959.tb00467.x

Hassan, H, O.M. Suriya, Z.A. Al-Aseri, M. Hasan and N. Khalid et al., 2015. Feasibility of using Arabic Hospital Anxiety and Depression Scale (HADS) to assess anxiety and depression among patients attending Accident and Emergency at a University Hospital setting in Riyadh, Saudi Arabia. Park J. Med. Sci., 31: 1366-1371.

Hung, M., J. Bounsanga, P. Tang, W. Chen and C. Cheng, 2015. The factor structure of the hospital anxiety and depression scale in orthopedic trauma patients. J. Clin. Med. Res., 7: 453-459. DOI: $10.14740 /$ jocmr2140w

Ishikawa, H., N. Kawakami and R.C. Kessler, 2015. Lifetime and 12-month prevalence severity and unmet need for treatment of common mental disorders in Japan: Results from the final dataset of World mental health Japan Survey. Epidemiol. Psychiatr.

Karam, E., Z. Mneimneh, H. Dimassi, J. Fayyad and A. Karam et al., 2008. Lifetime prevalence of mental disorders in Lebanon: First onset, treatment and exposure to war. PLos Med., 5: e61-e61. DOI: 10.1371/journal.pmed.0050061

Kawakami, N., E.A. Abdulghani, J. Alonso, E.J. Bromet and R. Bruffaerts et al., 2012. Early-life mental disorders and adult household income in the world mental health surveys. Bio. Psychiatry, 72: 228-237. DOI: 10.1016/j.biopsych.2012.03.009 
Kenny, DA., 2011. Respecification of latent variable models. http://davidakenny.net/cm/respec.htm

Kenny, DA., 2015. Measuring model fit. http://davidakenny.net/cm/fit.htm

Kersting, A., M. Dorsch, U. Wesselmann, K. Lüdorff and J. Witthaut et al., 2004. Maternal posttraumatic stress response after the birth of a very low-birthweight infant. J. Psychiatry Res., 57: 473-476.

Kessler, R.C. and P.S. Wang, 2008. The descriptive epidemiology of commonly occurring mental disorders in the United States. Ann. Rev. Public Health, 29: 115-129.

DOI: 10.1146/annurev.publhealth.29.020907.090847

Kessler, R.C., P. Berglund, O. Demler, R. Jin and K.R. Merikangas et al., 2005. Lifetime prevalence and age-of-onset distributions of DSM-IV disorders in the National Comorbidity Survey Replication. Archives General Psychiatry, 62: 593-602. DOI: 10.1001/archpsyc.62.6.593

Kummer, A., F. Cardoso and A.L. Teixeira, 2010. Generalized anxiety disorder and the Hamilton Anxiety Rating Scale in Parkinson's disease. Arquivos Neuro-Psiquiatria, 68: 495-501. DOI: $10.1590 / \mathrm{S} 0004-282 \mathrm{X} 2010000400005$

Leentjens, A.F., K. Dujardin, L. Marsh, P. MartinezMartin and I.H. Richard et al., 2011. Symptomatology and markers of anxiety disorders in Parkinson's disease: A cross-sectional study. Mov. Disord., 26: 484-92. DOI: 10.1002/mds.23528

Leentjens, A.F., K. Dujardin, L. Marsh, P. MartinezMartin and I.H. Richard et al., 2008. Anxiety rating scales in Parkinson's disease: Critique and recommendations. Mov. Disord., 23: 2015-2025. DOI: $10.1002 / \mathrm{mds} .22233$

Leichsenring, F., 2006. Cognitive-behavioral therapy and psychodynamic psychotherapy: Techniques, efficacy and indications. Am. J. Psychiatry, 60: 233-233.

Lenze, E. and J. Wetherell, 2011. A lifespan view of anxiety disorders. Dialogues Clin. Neuroscience, 13: 381-399.

Levinson, D., M.D. Lakoma, M. Petukhova, M. Schoenbaum and A.M. Zaslaysky et al., 2010. Associations of serious mental illness with earnings: Results from the WHO world mental health surveys. Br. J. Psychiatry, 197: 114-121.

DOI: 10.1192/bjp.bp.109.073635

Litster, B., K.M. Fiest, S.B. Patten, J.D. Fisk and J.R. Walker et al., 2016. Screening tools for anxiety in people with multiple sclerosis. Int. J. MS. Care, 18: 273-281. DOI: 10.7224/1537-2073.2016-004
Maier, W., R. Buller, M. Philipp and I. Heuser, 1988. The Hamilton anxiety scale: Reliability, validity and sensitivity to change in anxiety and depressive disorders. J. Affective Disorders, 14: 61-68. DOI: 10.1016/0165-0327(88)90072-9

Mondolo, F., M. Jahanshahi, A. Grana, E. Biasutti and E. Cacciatori et al., 2006. The validity of the hospital anxiety and depression scale and the geriatric depression scale in Parkinson's disease. Behav. Neurol., 17: 109-115. DOI: 10.1155/2006/136945

Remes, O., C. Brayne, R. van der Linde and L. Lafortune, 2016. A systematic review of reviews on the prevalence of anxiety disorders in adult populations. Brain Behav., 6: e00497-e00497. DOI: $10.1002 /$ brb3.497

Scott, J., A. Spector, M. Orrell, K. Scior and J. Sweeney et al., 2017. Limited validity of the Hospital Anxiety and Depression Scale (HADS) in dementia: Evidence from a confirmatory factor analysis. Int. J. Geriatr Psychiatry, 32: 805-813. DOI: $10.1002 /$ gps.4530

Scott, K.M., J.E. Wells, M. Angermeyer, T.A. Brugha and E. Bromet et al., 2010. Gender and the relationship between marital status and first onset of mood, anxiety and substance use disorders. Psychiatry Med., 40: 1495-1505.

Simpson, H.B., Y. Neria, R. Lewis-Fernandez and F. Schneier, 2010. Anxiety Disorders-Theory, Research and Clinical Perspectives. 1st Edn., Cambridge University Press, Cambridge.

Tanios, C.Y., M.T. Abou-Saleh, A.N. Karam, M.M. Salamoun and Z.N. Mneimneh et al., 2009. The epidemiology of anxiety disorders in the Arab world: A review. J. Anxiety Disord., 23: 409-419. DOI: $10.1016 /$ j.janxdis.2008.10.009

Terkawi, A., S. Tsang, G.J. Alkahtani, S.H. Al-Mousa and S. Al Misaed et al., 2017. Development and validation of Arabic version of the Hospital Anxiety and Depression Scale. Saudi J. Anaesth, 11: S11-S18. DOI: $10.4103 /$ sja.SJA_43_17

Thompson, E., 2015. Hamilton Rating Scale for Anxiety (HAM-A). Occupat. Med., 65: 601-601. DOI: $10.1093 /$ occmed/kqv054

Wang, J. and X. Wang, 2012. Structural Equation Modelling: Applications Using Mplus. 1st Edn., Wiley, Chichester, UK.

Yang, J., Y. Yin, C. Svob, J. Long and X. He et al., 2017. Amygdala atrophy and its functional disconnection with the cortico-striatal-pallidalthalamic circuit in major depressive disorder in females. PLoS One.

DOI: 10.1371/JOURNAL.PONE.0168239 